\title{
BINARITY AMONG BARIUM DWARFS AND CH SUBGIANTS: WILL THEY BECOME BARIUM GIANTS?
}

\author{
PIERRE NORTH \\ Institut d'Astronomie de l'Université de Lausanne \\ CH-1290 Chavannes-des-Bois, Switzerland \\ ALAIN JORISSEN \\ Institut d'Astronomie et d'Astrophysique \\ Université Libre de Bruxelles \\ B-1050 Bruxelles, Belgium \\ AND \\ MICHEL MAYOR \\ Observatoire de Genève \\ CH-1290 Sauverny, Switzerland
}

\begin{abstract}
We report the results of monitoring the radial velocities of 31 confirmed $\mathrm{Ba}$ dwarfs and $\mathrm{CH}$ subgiants, showing that about 27 of them have a variable radial velocity. Therefore, mass transfer in a binary most probably explains their chemical anomalies, as for the Ba giants. The orbital parameters of the 14 systems having sufficient phase coverage are very similar to those of the $\mathrm{Ba}$ giants. Whether the $\mathrm{CH}$ subgiants and $\mathrm{Ba}$ dwarfs may become $\mathrm{Ba}$ giants is discussed briefly. Most of the marginal $\mathrm{Ba}$ dwarfs listed by Edvardsson et al. (1993) are single, so that their chemical peculiarity, if real, cannot be due to binary evolution.
\end{abstract}

\section{Introduction}

The first barium stars discovered were all giants. The $\mathrm{CH}$ giants seem to be the metal-poor analogs of the Ba giants. Later on, Bond (1974) discovered what he called the $\mathrm{CH}$ subgiants, which have chemical anomalies very similar to those of the $\mathrm{Ba}$ and $\mathrm{CH}$ giants but are less evolved. Luck \& Bond (1982) recognized that some $\mathrm{CH}$ subgiants at least belong to the main sequence, according to the high surface gravities obtained from highresolution spectroscopic analyses. This became even clearer when Tomkin 
et al. (1989) discovered the F5 V star HR 107 to be a mild Ba star. Since then, still other main sequence $\mathrm{Ba}$ stars have been identified, so that the designation "Ba dwarf" seems justified and will be used here as an equivalent to "CH subgiant".

The questions this work tries to answer are quite simple:

- Are the Ba dwarfs the result of a mass-transfer event, like their giant counterparts?

- If so, is there an evolutionary link between them and the Ba giants? In other words, will the Ba dwarfs evolve into Ba giants?

A radial-velocity survey of 51 candidate and confirmed $\mathrm{Ba}$ dwarfs has been done in order to answer these questions.

\section{The Sample}

We have gathered our stars from a variety of sources, so that some of them are confirmed Ba dwarfs while others are not. Many have published abundances derived from high-resolution observations; those which did not were observed by us in the $\lambda 5100 \AA$ region to check their overabundances of $s$-process elements. However, a detailed abundance analysis of the latter is not yet available. We have divided our sample into two parts, according to the strength of their peculiarity:

1. 31 "strong" Ba dwarfs, confirmed by high-resolution spectroscopy.

These had been selected from the following sources:

- 9 stars from Bidelman $(1981,1983,1985)$ and confirmed as Ba dwarfs by North et al. (1994).

- 6 Ba stars with a spectral type earlier than G2 from the extensive list of Lü et al. (1983), which contains essentially Ba giants; the early spectral type was considered as betraying their dwarf rather than giant nature. Some of them have been confirmed by North et al. (1994) and by Luck \& Bond (1991).

- $5 \mathrm{~F}$ stars having a remark of the kind "Strong $\mathrm{Sr} 4077$ " in the Michigan catalogue (Houk \& Cowley 1975), and confirmed by us.

- 1 F star, HR 107, from Tomkin et al. (1989).

- $10 \mathrm{CH}$ subgiants from Luck \& Bond (1991).

2. 20 mild or marginal $\mathrm{Ba}$ dwarfs, including

- 10 candidates from Lü (1991), which were classified on the basis of low-resolution spectra ( $43 \AA \mathrm{mm}^{-1}$ ) as having a very slight Ba anomaly: $\mathrm{Ba} \leq 1$ on the usual scale running from 1 to 5 (Warner 1965). Our attempts to confirm them with high-resolution spectroscopy have failed, indicating that these stars cannot be considered real $\mathrm{Ba}$ dwarfs. However, we consider them here a useful 
comparison sample, since radial velocity measurements have been done for them as well.

- 9 mild Ba dwarfs from Edvardsson et al. (1993). These objects have typically $[s / \mathrm{Fe}] \approx 0.2$ and are therefore quite marginal.

- 1 candidate from Houk \& Cowley (1975), HD 9529, in which yttrium seems slightly overabundant, judging from the ratio of the lines Y II $\lambda 5087.43 / \mathrm{Fe}$ I $\lambda 5090.78$.

The spectral types range from about F3 to K5 for the whole sample, but most objects have types in the interval F5 - G2. The CH subgiants already monitored by McClure (1985) have not been included in our sample, except for HD 182274 which had been selected on the basis of its classification "F str $\lambda 4077$ " by Bidelman (1985).

\section{Observations}

Most radial velocities have been obtained using CORAVEL scanners (Baranne et al. 1979) attached, in the northern hemisphere, to the 1-m Swiss telescope at Observatoire de Haute-Provence, France, and in the southern hemisphere to the 1.54-m Danish telescope at ESO, La Silla, Chile. The measurements of the stars selected from Bidelman's papers began as early as 1987, even before their Ba nature was suspected, while the other stars, most of which can only be observed from the South, have been measured not earlier than 1992.

High-resolution spectra have been taken with the CES spectrograph fed by the 1.4-m CAT telescope at ESO in 1989, 1990, 1992 and/or 1993; the Short Camera was used, together with the RCA CCD \#9 (15 $\mu$ m pixels), the resolving power being 60000 in most cases. Although the primary goal was to obtain equivalent widths and abundances, good-quality radial velocities could be obtained as well, as a by-product. They have an accuracy comparable to that of CORAVEL, even if no radial-velocity standard were measured, thanks to the high resolution and excellent stability of the instrument.

\section{Results}

The rate of binaries we obtain is given below for the different categories of stars described above. In each case, we have made an estimate of the detection rate by generating artificial binaries at random, and "observing" them at the same epochs as the real ones, and with the same random error. Orbital elements typical of the giant Ba stars were assumed, as well as a mass of the secondary of $0.6 M_{\odot}$. 


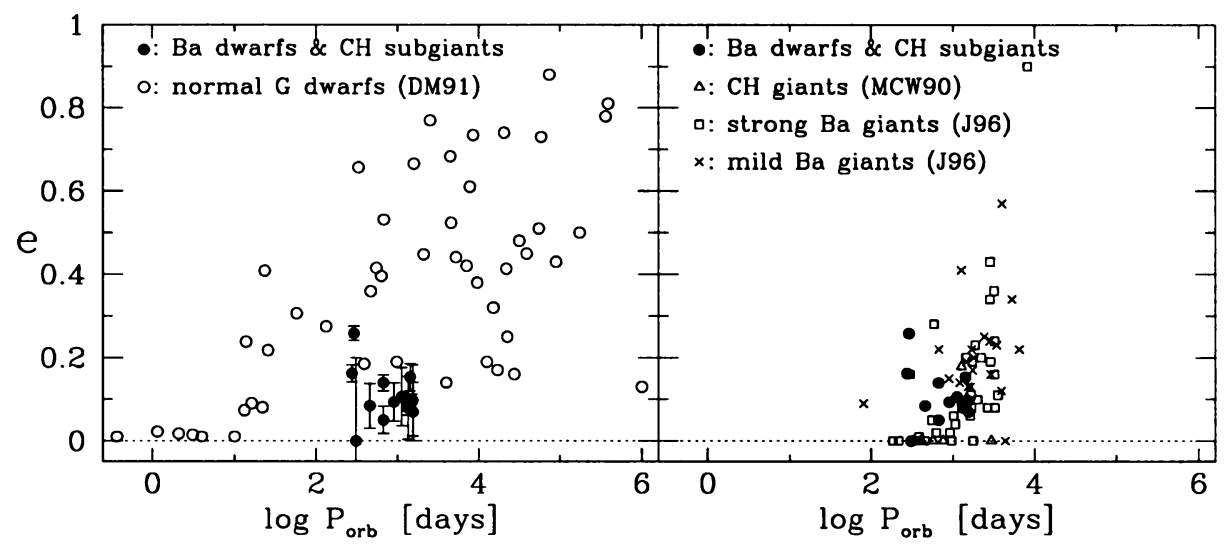

Figure 1. Left: $\mathrm{e}-\log \mathrm{P}$ diagram for the 14 orbits of Ba dwarfs (black dots) compared with the orbits of normal $\mathrm{G}$ dwarfs (open dots). Right: Comparison between Ba dwarfs and $\mathrm{Ba}$ giants, at the same scale; notice the bias against long periods for the dwarfs.

- Among the 10 candidates of Lü (1991), only 3 are binaries; one of these is an SB2 system, and the other two are SB1 binaries, one of which is only marginally detected.

- Among the 10 mild Ba dwarfs of Edvardsson et al. (1993) and HD 9529, we find only one SB1 system. Taking together these stars and Lü's, one obtains a rate of binaries of only $20 \%$, while the detection rate is better than $98 \%$. Therefore, the rate of binaries here is typical of normal rather than $\mathrm{Ba}$ stars.

- Among the 31 "strong" Ba dwarfs, we have found 28 binaries, one of which, HD 26455, is an SB2 system. Unfortunately, we do not have enough data to tell whether there is a third companion (the expected white dwarf) in this system or not. Excluding this star from the sample leads to a rate of $90 \%$, while the detection probability is $99 \%$.

Among the binaries, a very interesting system is HD 48565, which has the shortest period known among all Ba stars (giants and dwarfs): only 73 days, which is too short to allow the former primary to reach the typical radius of an AGB star. Recent measurements have shown a clear trend of the $\mathrm{O}-\mathrm{C}$ residuals, betraying the presence of a third component which is very probably the white dwarf. Since the short period is probably not linked with the WD but rather with some faint main-sequence star, we have not plotted this system on Figure 1.

Among the 28 binaries found in the sample of the 31 "strong" Ba dwarfs, we could determine only 14 orbits, all of which have long periods and small eccentricities, as shown in Figure 1. The binaries among normal G dwarfs studied by Duquennoy \& Mayor (1991) are shown for comparison. The 


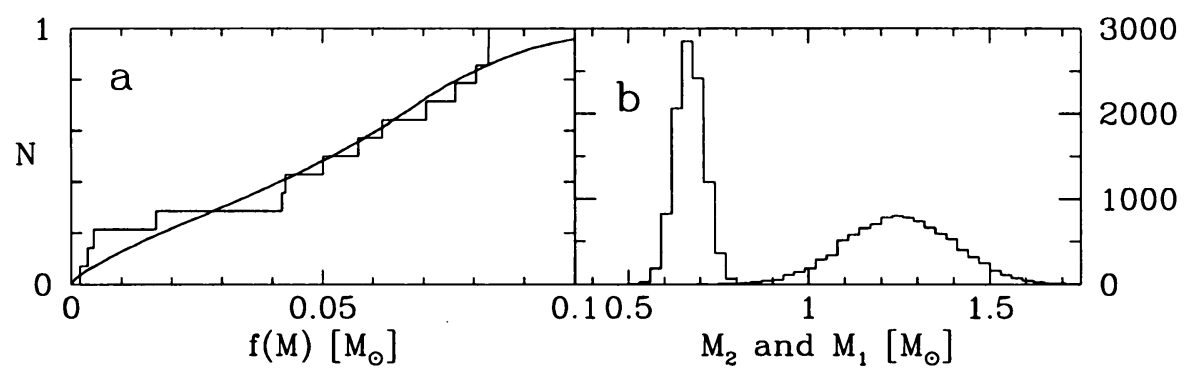

Figure 2. (a) Observed and simulated cumulative distribution of the mass functions. (b) Gaussian distributions of the masses of the secondary (left) and of the primary (right) used in the simulation. The distribution centered on $1.25 M_{\odot}$ reproduces approximately the observed one.

contrast is striking: some dissipation mechanism must have acted upon the orbits of the $\mathrm{Ba}$ dwarfs to reduce their excentricity. On the other hand, the orbital elements of the Ba dwarfs are very similar to those of the Ba giants, although the former suffer from a bias against long periods.

A very interesting possibility offered by the $\mathrm{Ba}$ dwarfs is that of a direct, though statistical, estimate of the average mass of their companion. Contrary to the case of the Ba giants, the mass of the primary can be determined using a photometric estimate of the effective temperature and spectroscopic estimates of the surface gravity and metallicity. An interpolation in the evolutionary tracks of Schaller et al. (1992), Schaerer et al. (1993) and Charbonnel et al. (1993) gives the mass. Then we compare the observed cumulative distribution of the mass functions with a simulated distribution, where we assume random orientations of the orbits and approximate the observed distribution of the primary's masses by a gaussian. The best fit, shown in Figure 2, occurs for an average secondary mass of

$$
M_{2}=0.67 \pm 0.09 M_{\odot}
$$

where the uncertainty corresponds to the $95 \%$ confidence level. This is the first direct estimate of the average mass of the companion in a sample of $\mathrm{Ba}$ stars. Our result is entirely compatible with the masses of white dwarfs resulting from 2-3 $M_{\odot}$ progenitors (Reid 1996).

\section{Conclusion}

We have shown to a high degree of probability that $\mathrm{Ba}$ dwarfs, like $\mathrm{Ba}$ giants, result from a mass-transfer event in a binary. Will they become $\mathrm{Ba}$ giants after they have left the main sequence? From the point of view of the abundances, the answer is yes, according to Smith et al. (1993). From the "orbital" point of view, the answer is yes for the most massive dwarfs of our 
sample, but no for the others. Indeed, the mass functions of the Ba dwarfs are much larger than those of the $\mathrm{Ba}$ giants, and are closer to those of the $\mathrm{CH}$ giants. Therefore the $\mathrm{Ba}$ giants are statistically more massive than the $\mathrm{Ba}$ dwarfs, consistent with the estimate of $1.65 M_{\odot}$ (instead of $\sim 1.25$ $M_{\odot}$ for the dwarfs) by Jorissen et al. (1998). But this difference might be due to a detection bias against massive $\mathrm{Ba}$ dwarfs: the Am phenomenon is frequently present for spectral types A5-F0 and includes overabundances of $s$-process elements, but due to radiative diffusion (Michaud 1991). Perhaps some massive $\mathrm{Ba}$ dwarfs are concealed among $\mathrm{Am}-\mathrm{Fm}$ stars.

\section{References}

Baranne, A., Mayor, M. \& Poncet, J.L. 1979, Vistas in Astron., 23, 279

Bidelman, W.P. 1981, $A J, 86,553$

Bidelman, W.P. 1983, $A J, 88,1182$

Bidelman, W.P. 1985, $A J, 90,341$

Bond, H. E. 1974, ApJ, 194, 95

Charbonnel, C., Meynet, G., Maeder, A., Schaller, G. \& Schaerer, D. 1993, A\&A Supp., 101,415

Duquennoy, A. \& Mayor, M. 1991, $A \& A, 248,485$

Edvardsson, B., Andersen, J., Gustafsson, B., Lambert, D. L., Nissen, P.E. \& Tomkin, J. 1993, $A \& A, 275,101$

Houk, N. \& Cowley, A.P. 1975, University of Michigan Catalogue of Two-Dimensional Spectral Types for the HD Stars, Vol. 1, University of Michigan, Ann Arbor

Jorissen, A., Van Eck, S., Mayor, M. \& Udry, S. 1998, $A \& A$, 332, 877

Lü, P. K. 1991, $A J, 101,2229$

Lü, P. K., Dawson, D. W., Upgren, A. R. \& Weis, E. W. 1983, ApJ Supp., 52, 169

Luck, R.E. \& Bond, H.E. 1982, ApJ, 259, 792

Luck, R. E. \& Bond, H. E. 1991, ApJ Supp., 77, 515

McClure, R. D. 1985, in Cool Stars with Excesses of Heavy Elements, ed. M. Jaschek and P. C. Keenan (Reidel), p. 159

Michaud, G. 1991, in IAU Symp. 145: Evolution of Stars: The Photospheric Abundance Connection, ed. G. Michaud and A. Tutukov (Kluwer), p. 111

North, P., Berthet, S. \& Lanz, T. 1994, $A \& A, 281,775$

Reid, I. N. 1996, $A J, 111,2000$

Schaller, G., Schaerer, D., Meynet, G. \& Maeder, A. 1992, A\&A Supp., 96, 269

Schaerer, D., Meynet, G., Maeder, A. \& Schaller, G. 1993, A\&A Supp., 98, 523

Smith, V.V., Coleman, H. \& Lambert, D. L. 1993, ApJ, 417, 287

Tomkin, J., Lambert, D. L., Edvardsson, B., Gustafsson, B. \& Nissen, P. E. 1989, $A \& A$, 219, L15

Warner, B. 1965, MNRAS, 129, 263 


\section{Discussion}

Feast: What constraints do the relative space densities place on the question of whether or not $\mathrm{Ba}$ dwarfs evolve into variable giants?

North: The frequency of the $\mathrm{Ba}$ dwarfs belonging to binaries is about $1-2 \%$ of all mid-F to early $G$ dwarfs, which is roughly the same as the frequency of $\mathrm{Ba}$ giants among the $\mathrm{G}-\mathrm{K}$ giants. Therefore it is reasonable to assume that $\mathrm{Ba}$ dwarfs evolve into $\mathrm{Ba}$ giants.

Little-Marenin: What is the origin of the non-binary mild Ba dwarfs?

North: I am not sure; possibly primordial.

Jorissen: In a sample of 28 mild $\mathrm{Ba}$ giants ( $\mathrm{Ba} 1$ stars) monitored for about 10 years with CORAVEL, at least 3 (HD 50843, HD 95345 and HD $119185)$ do not seem to be binaries. Sneden et al. (1981, $A p J, 247,1052)$ analyzed HD 95345, showing that it is definitely a barium star, though with moderate overabundances. Galactic fluctuations in the primordial $\mathrm{Ba} / \mathrm{Fe}$ ratio may perhaps account for this small fraction $(\sim 10 \%)$ of non-binary mild Ba stars.

Gustafsson: One should, of course, be careful when comparing mainsequence Ba stars with Ba-rich giants, in particular when the previous ones are F-type dwarfs having comparatively shallow convection zones. It may be easy to pollute one of those, but hard to keep this pollution visible when the convection zone deepens and includes more mass. I presume the precursors of the $\mathrm{Ba}$ giants must have been lower-mass dwarfs with deep convection zones which, by accretion, have moved up along the main sequence. Alternatively, other mixing processes were active at the mass transfer.

North: The abundance anomalies do not necessarily remain confined within the outer convective zone. On the contrary, the $\mu$ inversion implies a mixing in the radiative zone, and on a rather short timescale, as shown by Proffitt \& Michaud (1989, ApJ, 345, 998). 